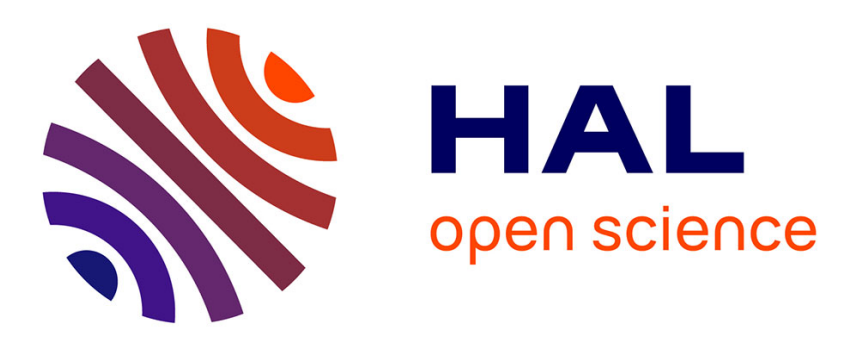

\title{
Calcul sur ordinateur de champ et de flux magnétique de bobines circulaires et de cadres rectangulaires
}

\author{
P. Bois, M. La Porte, D. Mace, M. Waligora
}

\section{To cite this version:}

P. Bois, M. La Porte, D. Mace, M. Waligora. Calcul sur ordinateur de champ et de flux magnétique de bobines circulaires et de cadres rectangulaires. Revue de Physique Appliquée, 1972, 7 (4), pp.223-233. 10.1051/rphysap:0197200704022300 . jpa-00243623

\section{HAL Id: jpa-00243623 https://hal.science/jpa-00243623}

Submitted on 1 Jan 1972

HAL is a multi-disciplinary open access archive for the deposit and dissemination of scientific research documents, whether they are published or not. The documents may come from teaching and research institutions in France or abroad, or from public or private research centers.
L'archive ouverte pluridisciplinaire HAL, est destinée au dépôt et à la diffusion de documents scientifiques de niveau recherche, publiés ou non, émanant des établissements d'enseignement et de recherche français ou étrangers, des laboratoires publics ou privés. 


\title{
CALCUL SUR ORDINATEUR DE CHAMP ET DE FLUX MAGNÉTIQUE DE BOBINES CIRCULAIRES ET DE CADRES RECTANGULAIRES
}

\author{
P. BOIS, M. LA PORTE, D. MACE et M. WALIGORA \\ Institut Français du Pétrole, 4, avenue de Bois-Préau, 92-Rueil-Malmaison
}

(Reçu le 16 décembre 1971, révisé le 2 mai 1972)

\begin{abstract}
Résumé. - L'utilisation des bobines étant devenue de plus en plus fréquente, nous avons pensé qu'il serait utile de communiquer certains sous-programmes écrits en langage Fortran. Ils permettent de calculer le champ magnétique créé par une bobine circulaire épaisse (BOB) ou par une bobine circulaire mince (BOB2) ou encore par un cadre mince rectangulaire (HCADRE). Deux autres sous-programmes calculent le flux magnétique créé par un aimant élémentaire et traversant un cadre rectangulaire mince (FLUXA et FLUXB). En outre figurent également trois sous-programmes de service : calcul des intégrales définies par la méthode du pas variable automatique (INTEGR), calcul des intégrales elliptiques complètes de première et deuxième espèce (ECF et ECE). Pour chaque sous-programme, nous donnons le mode d'emploi, ainsi que la formulation algèbrique dont il est la traduction.
\end{abstract}

\begin{abstract}
The use of coils becoming more and more frequent, we thought it useful to give some subroutines written in the Fortran Language. These programs make it possible to calculate the magnetic field created either by a thick circular coil (BOB) or by a thin circular coil (BOB2) or by a rectangular thin frame (HCADRE). Two other subroutines calculate the magnetic flux generated by a magnetic dipole and crossing a magnetic thin frame (FLUXA and FLUXB). Besides these, three other subroutines are given : calculation of definite integrals by the method of automatically variable steps (INTEGR). Calculation of complete elliptic integrals of first and second kind (ECF and ECE). For every subroutine, we give instructions for use and formulation.
\end{abstract}

1. Introduction. - Ces dernières années, un très grand nombre de magnétomètres à induction avec ou sans noyau de $\mu$ métal ont été construits ainsi que de nombreux solénoïdes, bobines ou cadres, ces derniers pour l'étude de différents champs magnétiques. A l'institut Français du Pétrole, nous avons été amenés, lors d'études sur la résonance magnétique nucléaire à utiliser des bobines et à déterminer leurs champs et flux magnétiques. Ces caractéristiques peuvent être mesurées expérimentalement, mais les méthodes utilisées sont peu précises et la mise en œuvre est longue. Nous avons préféré écrire en langage Fortran une série de sous-programmes. Ils permettent de calculer les champs et flux magnétiques de bobines circulaires et de cadres rectangulaires. Nous avons pensé que ces sous-programmes pourraient intéresser tous les utilisateurs de solénoïdes, de cadres rectangulaires ou de magnétomètres; aussi nous donnons, dans ce qui suit, le mode d'emploi de chacun de ces sous-programmes ainsi que la formulation qui a servi à les élaborer. Ces sous-programmes spécialisés font intervenir trois sous-programmes de service : calcul des intégrales définies par la méthode du pas variable automatique (INTEGR); calcul des intégrales elliptiques complètes de première et deuxième espèce (ECF et ECE). Nous donnons également deux illustrations graphiques : l'une représentant les lignes de force du champ d'une bobine mince et l'autre représentant des lignes d'égale valeur du champ d'une bobine mince.

2. Description des sous-programmes. - Les données fournies à ces différents sous-programmes ainsi que les résultats sont tous exprimés dans le système CGS. Nous donnons ci-dessous une fiche pour chaque sousprogramme.

\section{SOUS-PROGRAMME BOB}

But. - Le sous-programme BOB permet de calculer le champ magnétique créé par une bobine épaisse d'axe vertical en un point $M$ extérieur à cette bobine en fonction des données suivantes (Fig. 1) :

$-R_{0}$ rayon moyen $a$ de la bobine $(\mathrm{cm})$.

- $C$ épaisseur de la bobine $(\mathrm{cm})$.

$-Z_{1}, Z_{2}$ cotes des faces inférieure $\left(Z_{1}\right)$ et supérieure $\left(Z_{2}\right)$ de la bobine $(\mathrm{cm})$.

- AMP intensité $I$ du courant (ampères).

AMP $>0$, si le courant est en sens inverse des aiguilles d'une montre.

AMP $<0$, si le courant est dans le sens des aiguilles d'une montre.

- AN nombre total $N$ de spires.

- $X, Y, Z$ coordonnées du point $\mathrm{M}$ où l'on calcule le champ magnétique $(\mathrm{cm})$. 


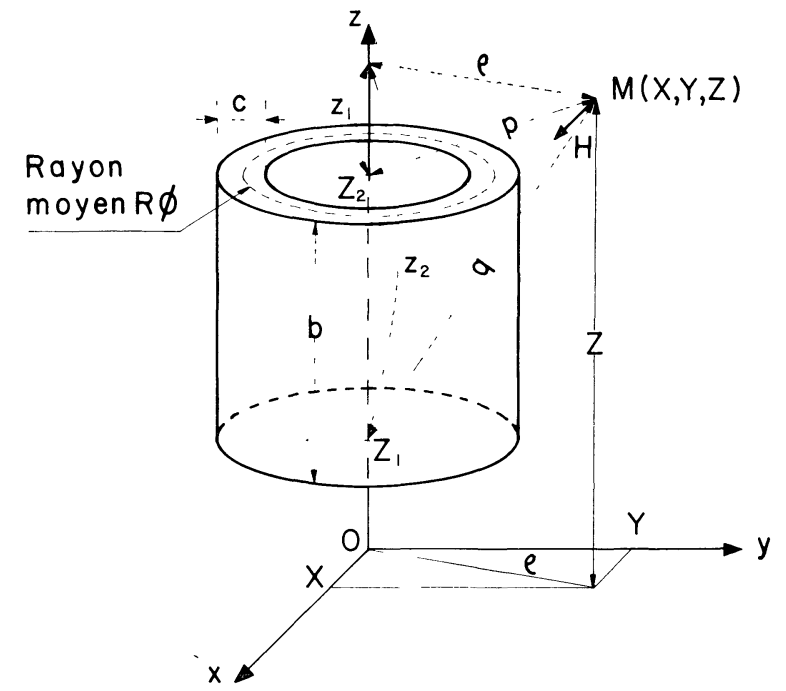

FIG. 1. - Le sous-programme BOB calcule le champ magnétique créé par une bobine épaisse d'axe vertical $\mathrm{O} z$ en un point extérieur à cette bobine.

Le champ magnétique au point $\mathrm{M}$ est donné par ses trois composantes sur les axes $x, y, z: H_{x}, H_{y}, H_{z}$ exprimées en œrsteds.

Remarque. - Un diagnostic ECHEC permet de savoir si les données sont bien conformes aux hypothèses $\mathrm{du}$ programme :

si $\mathrm{ECHEC}=0$. Le calcul se déroule normalement; si $\mathrm{ECHEC}=1$. Le calcul a échoué (voir Chap. suivant).

Formulation. - Ce sous-programme BOB utilise les formules exposées par H. B. Dwight dans un article intitulé «The magnetic field of a circular cylindrical coil » publié dans the London, Edinburgh and Dublin Philosophical Magazine and Journal of Science, d'avril 1931.

Nous donnons ci-dessous très brièvement les notations et les formules de H. B. Dwight

$$
\begin{aligned}
& z_{1}=Z-Z_{2}, \\
& z_{2}=Z-Z_{1}, \\
& b=Z_{2}-Z_{1} \text { (longueur de la bobine) } \\
& \rho=\left(X^{2}+Y^{2}\right)^{1 / 2} \\
& p=\left(z_{1}^{2}+\rho^{2}\right)^{1 / 2} \\
& q=\left(z_{2}^{2}+\rho^{2}\right)^{1 / 2} \\
& \mu_{1}=\frac{z_{1}}{p} \\
& \mu_{2}=\frac{z_{2}}{q} .
\end{aligned}
$$

En considérant l'expression :

$$
V(n)=\left\{\left(1+\frac{c}{2 a}\right)^{n}-\left(1-\frac{c}{2 a}\right)^{n}\right\} \frac{a}{n c},
$$

la composante axiale $F \mathrm{du}$ champ en un point $\mathrm{M}$ s'écrit sous la forme de la série suivante :

$$
\begin{gathered}
F=\frac{\pi N I}{10 b} \sum_{j=1}^{\infty}(-1)^{j+1} \frac{(2 j) !}{(j !)^{2} 2^{2 j-1}} \times \\
\times\left\{\left(\frac{a}{p}\right)^{2 j} P_{2 j-1}\left(\mu_{1}\right)-\left(\frac{a}{q}\right)^{2 j} P_{2 j-1}\left(\mu_{2}\right)\right\} V(2 j+1),
\end{gathered}
$$

où les polynômes $P_{j}(\mu)$ sont donnés par la relation de récurrence suivante :

$$
(j+1) P_{j+1}(\mu)=(2 j+1) \mu P_{j}(\mu)-j P_{j-1}(\mu),
$$

avec $P_{0}(\mu)=1$ et $P_{1}(\mu)=\mu$.

La composante radiale $R$ du champ en un point $\mathrm{M}$ est également la somme d'une série :

$$
\begin{aligned}
R=\frac{\pi N I \rho}{10 a b} \sum_{j=1}^{\infty}(-1)^{j+1} \frac{(2 j) !}{(j !)^{2}(2 j-1) 2^{2 j-1}} \times \\
\quad \times\left\{\left(\frac{a}{p}\right)^{2 j+1} P_{2 j-1}^{\prime}\left(\mu_{1}\right)-\left(\frac{a}{q}\right)^{2 j+1} P_{2 j-1}^{\prime}\left(\mu_{2}\right)\right\} \\
\quad \times V(2 j+1)
\end{aligned}
$$

où les polynômes $P_{j}^{\prime}(\mu)$ sont donnés par la relation de récurrence suivante :

$$
\left(\mu^{2}-1\right) P_{j}^{\prime}(\mu)=j \mu P_{j}(\mu)-j P_{j-1}(\mu)
$$

avec $P_{0}^{\prime}(\mu)=0$.

Pour que le calcul du champ puisse s'exécuter correctement en machine, il faut que la série soit rapidement convergente. Ceci entraîne un certain nombre de contraintes sur les valeurs des paramètres de la bobine :

- le rayon moyen $a$ doit être bien inférieur aux quantités $p$ et $q$,

- $z_{1}$ et $z_{2}$ doivent être bien inférieurs respectivement aux quantités $p$ et $q$.

Si les données fournies au programme ne sont pas satisfaisantes, le développement en série ne converge pas et un diagnostic nous est fourni sous la forme de $\mathrm{ECHEC}=1$.

\section{SOUS-PROGRAMME BOB 2}

But. - Le sous-programme BOB2 permet de calculer le champ magnétique créé par une bobine mince d'axe vertical en tout point $M$ extérieur ou intérieur à cette bobine, en fonction des données suivantes (Fig. 2) :

- $R$ rayon de la bobine $(\mathrm{cm})$.

$-Z_{1}, Z_{2}$ cotes des iaces inférieure et supérieure de la bobine $(\mathrm{cm})$.

- AMP intensité du courant (ampères). 


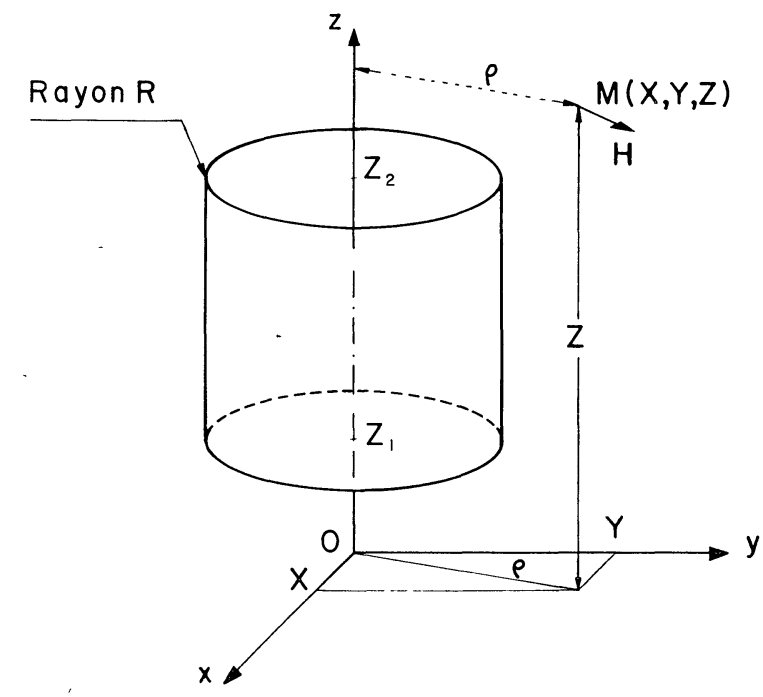

FIG. 2. - Le sous-programme BOB2 calcule le champ magnétique créé par une bobine mince d'axe vertical en un point intérieur ou extérieur à cette bobine.

AMP $>0$, si le courant est en sens inverse des aiguilles d'une montre.

AMP $<0$, si le courant est dans le sens des aiguilles d'une montre.

- AN nombre total de spires.

$-X, Y, Z$ coordonnées du point $\mathrm{M}$ où l'on calcule le champ magnétique $(\mathrm{cm})$.

Le champ magnétique au point $M$ est donné par ses trois composantes sur les axes $x, y, z: H X, H Y$, $H Z$ exprimées en œrsteds.

Formulation. - La composante radiale $H_{\rho} \mathrm{du}$ champ magnétique en un point $\mathrm{M}$ est donnée par:

$$
\begin{aligned}
H_{\rho}=\frac{0,4 \times R \times \mathrm{AMP}}{Z_{2}-Z_{1}} & \times \mathrm{AN} \\
& \times \frac{F\left(\lambda_{1}\right)-2 G\left(\lambda_{1}\right)}{\left[(R+\rho)^{2}+\left(Z-Z_{1}\right)^{2}\right]^{1 / 2}} \\
& \times \frac{F\left(\lambda_{2}\right)-2 G\left(\lambda_{2}\right)}{\left[(R+\rho)^{2}+\left(Z-Z_{2}\right)^{2}\right]^{1 / 2}}
\end{aligned}
$$

avec $\rho^{2}=X^{2}+Y^{2}$,

$$
\lambda_{1}=2\left[\frac{R \rho}{(R+\rho)^{2}+\left(Z-Z_{1}\right)^{2}}\right]^{1 / 2}
$$

et

$$
\lambda_{2}=2\left[\frac{R \rho}{(R+\rho)^{2}+\left(Z-Z_{2}\right)^{2}}\right]^{1 / 2} .
$$

Les fonctions $F(x)$ et $E(x)$ sont respectivement les intégrales elliptiques de première et seconde espèce

$$
G(x)=\frac{F(x)-E(x)}{x^{2}} .
$$

Ces différentes fonctions sont explicitées dans l'annexe (formules (VII), (VIII) et (IX)).

Les deux composantes horizontales du champ magnétique peuvent s'obtenir à partir de la composante radiale :

$$
\begin{aligned}
& H X=H_{\rho} \frac{X}{\rho} \\
& H Y=H_{\rho} \frac{Y}{\rho} .
\end{aligned}
$$

La composante verticale s'écrit :

$$
\begin{aligned}
& H Z=\frac{0,2 \times R \times \mathrm{AMP} \times \mathrm{AN}}{Z_{2}-Z_{1}} \int_{0}^{\pi} \frac{(R-\rho \cos \alpha)}{\left(R^{2}+\rho^{2}-2 R \rho \cos \alpha\right)}\left[\frac{Z-Z_{1}}{\left(R^{2}+\rho^{2}+\left(Z-Z_{1}\right)^{2}-2 \rho R \cos \alpha\right)^{1 / 2}}-\right. \\
& \left.-\frac{Z-Z_{2}}{\left(R^{2}+\rho^{2}+\left(Z-Z_{2}\right)^{2}-2 \rho R \cos \alpha\right)^{1 / 2}}\right] \mathrm{d} \alpha \text {. }
\end{aligned}
$$

Démonstration. - 1. CHAMP CRÉÉ EN UN POINT M DU PLAN $x O z$ PAR UN ÉLÉMENT DE SPIRE CIRCULAIRE HORIZONTALE (Fig. 3). - Nous savons que le champ créé en un point $\mathrm{M}$ par un élément de courant $i$ ds est donné par la formule de Laplace :

$$
\mathrm{d} \mathfrak{H}_{\mathrm{M}}=\frac{i}{10} \frac{\mathbf{d s} \wedge \mathbf{P M}}{r^{3}},
$$

avec longueur $=\mathrm{cm}$

intensité $=$ ampère

champ $=$ œrsted

si on pose $r^{2}(z)=\mathrm{PM}^{2}=x^{2}+z^{2}+R^{2}-2 x R \cos \alpha$.

Le champ magnétique créé en un point $M$ par un élément de spire circulaire horizontale est égal à :

$$
\mathrm{d}_{\mathscr{E}_{\mathrm{M}}} \mid \begin{array}{ll}
\frac{i}{10} & \frac{z R \cos \alpha \mathrm{d} \alpha}{r^{3}} \\
\frac{i}{10} & \frac{z R \sin \alpha \mathrm{d} \alpha}{r^{3}} \\
\frac{i}{10} & \frac{R(R-x \cos \alpha) \mathrm{d} \alpha}{r^{3}}
\end{array}
$$

2. CHAMP $\mathfrak{H}_{\mathrm{M}}$ CRÉÉ EN UN POINT M PAR LA SPIRE ENTIÈRE. - Ce champ est donné par :

$$
\mathfrak{H}_{\mathrm{M}}=\int_{\alpha=0}^{2 \pi} \mathrm{d} \mathfrak{H}_{\mathrm{M}} \text {. }
$$

Remarquons que la composante $\mathcal{H}_{y}$ est nulle car $\mathscr{H}_{\mathrm{M}}$ est dans le plan $x \mathrm{z}$. 


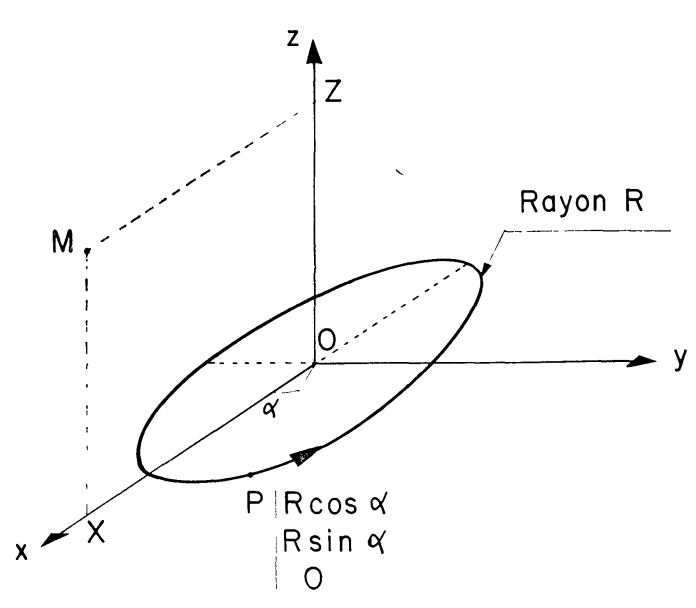

FIG. 3.

3. CHAMP $\mathbf{H}_{\mathbf{M}}$ CRÉÉ PAR UNE BOBINE EN UN POINT M DU PLAN $x \mathrm{O} z$. - Le point $\mathrm{M}$ a pour coordonnées $(\rho, \mathrm{O}, Z)$ avec $\rho \geqslant 0$. La densité de courant $\sigma$, à la surface de la bobine vaut:

$$
\sigma=\frac{\text { intensité } \times \text { nombre de spires }}{\text { longueur de la bobine }}=\frac{\mathrm{AMP} * \mathrm{AN}}{Z_{2}-Z_{1}} .
$$

La spire élémentaire, de cote $\zeta$ et l'épaisseur $\mathrm{d} \zeta$

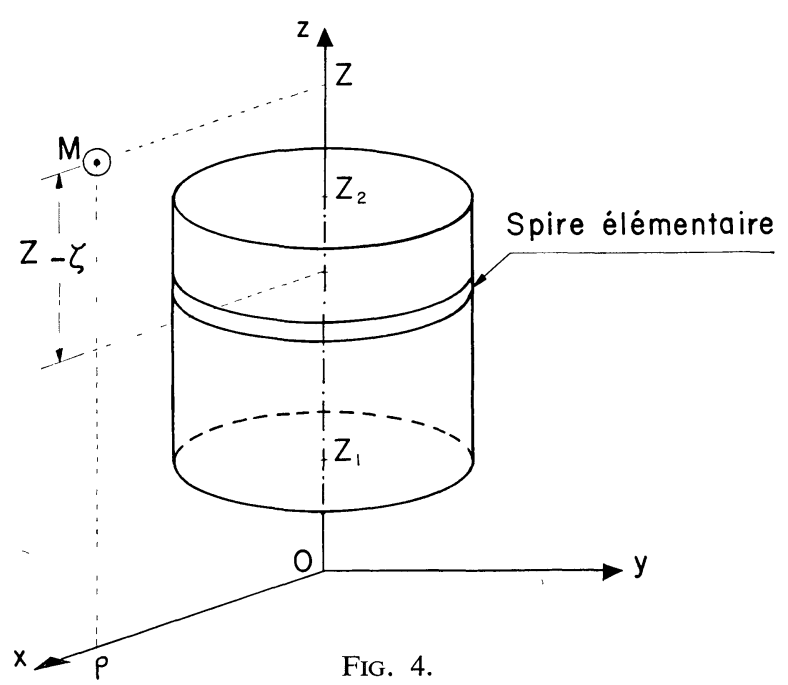

est parcourue par un courant $\sigma \mathrm{d} \zeta$. On a d'après la figure 4 :

$$
\begin{aligned}
z & =Z-\zeta, \\
\mathrm{d} z & =-\mathrm{d} \zeta .
\end{aligned}
$$

Ainsi l'intensité $i$ que nous trouvons dans l'expression de $\mathrm{d} \mathscr{H}_{\mathrm{M}}$ est égale à :

$$
i=\sigma \mathrm{d} \zeta=-\sigma \mathrm{d} z
$$

Composante horizontale (radiale)

$$
H_{\rho}=-\frac{R \sigma}{10} \int_{Z-Z_{1}}^{Z-Z_{2}} z \mathrm{~d} z \int_{0}^{2 \pi} \frac{\cos \alpha \mathrm{d} \alpha}{r^{3}}=\frac{R \sigma}{10} \int_{0}^{2 \pi} \cos \alpha \mathrm{d} \alpha\left|\frac{1}{r}\right|_{z=Z-Z_{1}}^{z=Z-Z_{2}}
$$

d'après la formule (I) de l'annexe.

$$
H_{\rho}=\frac{R \sigma}{10}\left|\frac{4\left\{2 G\left(\sqrt{\frac{4 R \rho}{(\rho+R)^{2}+z^{2}}}\right)-F\left(\sqrt{\frac{4 R \rho}{(\rho+R)^{2}+z^{2}}}\right)\right.}{\left[(\rho+R)^{2}+z^{2}\right]^{1 / 2}}\right|_{z=\mathrm{Z}-\mathrm{Z}_{1}}^{z=\mathrm{z}-\mathrm{z}_{2}}
$$

d'après la formule $(\mathrm{X})$ de l'annexe.

$$
H_{\rho}=0,4 R \sigma\left\{\frac{F\left(\lambda_{1}\right)-2 G\left(\lambda_{1}\right)}{\left[(\rho+R)^{2}+\left(Z-Z_{1}\right)^{2}\right]^{1 / 2}}-\frac{F\left(\lambda_{2}\right)-2 G\left(\lambda_{2}\right)}{\left[(\rho+R)^{2}+\left(Z-Z_{2}\right)^{2}\right]^{1 / 2}}\right\},
$$

avec :

$$
\lambda_{1}=\left[\frac{4 R \rho}{(\rho+R)^{2}+\left(Z-Z_{1}\right)^{2}}\right]^{1 / 2} \lambda_{2}=\left[\frac{4 R \rho}{(\rho+R)^{2}+\left(Z-Z_{2}\right)^{2}}\right]^{1 / 2} .
$$

Composante verticale

$$
H_{z}=-\frac{R \sigma}{10} \int_{Z-Z_{1}}^{Z-Z_{2}} \mathrm{~d} z \int_{0}^{2 \pi} \frac{(R-\rho \cos \alpha) \mathrm{d} \alpha}{r^{3}(z)}
$$

ce qui donne d'après la formule (II) de l'annexe :

$$
H_{z}=-\frac{R \sigma}{10} \int_{0}^{2 \pi} \frac{R-\rho \cos \alpha}{R^{2}+\rho^{2}-2 R \rho \cos \alpha}\left|\frac{z}{r(z)}\right|_{z=Z-Z_{1}}^{z=Z-Z_{2}} \mathrm{~d} \alpha
$$


Remarques. - Le calcul de $H Z$ fait appel au sousprogramme INTEGR qui fait partie de la bibliothèque des sous-programmes de l'IFP.

Les fonctions $F(\xi)$ et $E(\xi)$ sont calculées par les fonctions ECF et ECE qui font partie également de la bibliothèque des sous-programmes de l'IFP.

La fonction $G(\xi)=(F(\xi)-E(\xi)) / \xi^{2}$ vaut $\pi / 4$ lorsque $\xi=0$.

La formulation précédente est en défaut si l'on a simultanément :

$$
\rho=R \quad \text { et } \quad Z_{1} \leqslant Z \leqslant Z_{2}
$$

c'est-à-dire si le point se situe sur la bobine. On sait que le fait de traverser la bobine ne change pas la composante normale (radiale) mais change de $4 \pi \sigma$ la composante tangentielle (verticale). Pour éviter cet inconvénient, lorsque $\rho$ est très voisin de $R$, c'està-dire :

$$
\left|\frac{\rho-R}{R}\right|<0,001,
$$

le sous-programme modifie conventionnellement la valeur de $\rho$ et adopte suivant les cas :

$$
\text { soit } \rho=0,999 R, \quad \text { soit } \rho=1,001 R \text {. }
$$

Le calcul de $H_{z}$ s'obtient par une quadrature numérique effectuée à l'aide du sous-programme INTEGR. Cette technique est assez lourde. Il serait peut-être plus avantageux de passer par l'intermédiaire des intégrales elliptiques de troisième espèce, ce qui nécessiterait la création du sous-programme correspondant. Cette étude n'a pas été faite.

\section{SOUS-PROGRAMME HCADRE}

But. - Le sous-programme HCADRE permet de calculer le champ magnétique créé par un cadre mince, rectangulaire, vertical, en un point $\mathrm{M}$ en fonction des données suivantes (Fig. 5) :

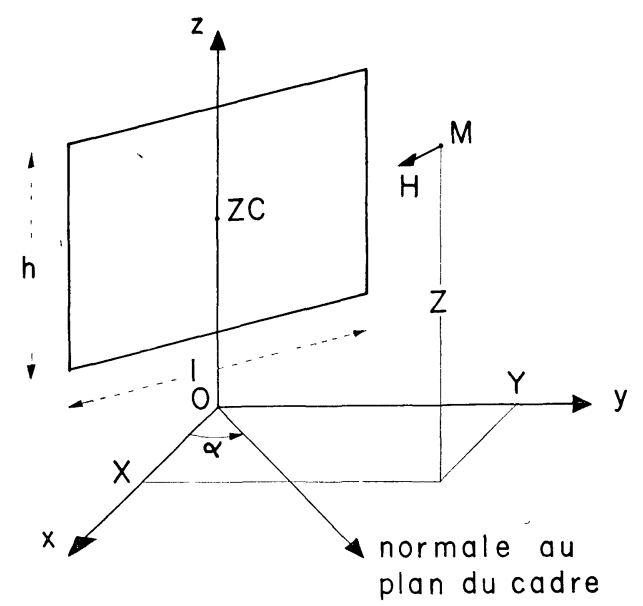

FIG. 5. - Le sous-programme HCADRE calcule le champ magnétique créé par un cadre mince vertical d'axe $\mathrm{O} z$ en un point quelconque de l'espace.
- CH la hauteur $h$ du cadre $(\mathrm{cm})$.

- CL la largeur $l$ du cadre $(\mathrm{cm})$.

- ZC la cote du centre du cadre $(\mathrm{cm})$.

- CI l'intensité $i$ du courant (ampère).

- $\mathrm{CN}$ nombre $n$ de spires.

- CA angle orienté $\alpha$ de Ox avec la normale positive au cadre (degrés).

- $X, Y, Z$ coordonnées du point $\mathbf{M}$ où on calcule le champ magnétique $(\mathrm{cm})$.

Le champ magnétique au point $\mathbf{M}$ est donné par ses trois composantes sur les axes, $x, y, z: H X, H Y$, $H Z$ exprimées en œrsteds.

Formulation. - Les formules utilisées dans ce sousprogramme pour donner les composantes du champ magnétique sont les suivantes:

- composante normale au cadre :

$$
\begin{aligned}
& H_{n}=\frac{i n}{10}\left[y_{1} \psi\left(z_{1}, z_{2}, y_{1}\right)-y_{2} \psi\left(z_{1}, z_{2}, y_{2}\right)+\right. \\
& \left.\quad+z_{1} \psi\left(y_{1}, y_{2}, z_{1}\right)-z_{2} \psi\left(y_{1}, y_{2}, z_{2}\right)\right]
\end{aligned}
$$

- composante horizontale dans le plan du cadre :

$$
H_{h}=\frac{i n}{10} x\left[\psi\left(z_{1}, z_{2}, y_{2}\right)-\psi\left(z_{1}, z_{2}, y_{1}\right)\right],
$$

- composante verticale

$$
H_{z}=\frac{i n}{10} x\left[\psi\left(y_{1}, y_{2}, z_{2}\right)-\psi\left(y_{1}, y_{2}, z_{1}\right)\right]
$$

avec $x=X \cos \alpha-Y \sin \alpha$

$$
\begin{aligned}
& y=X \sin \alpha+Y \cos \alpha \\
& z=Z-Z C
\end{aligned}
$$

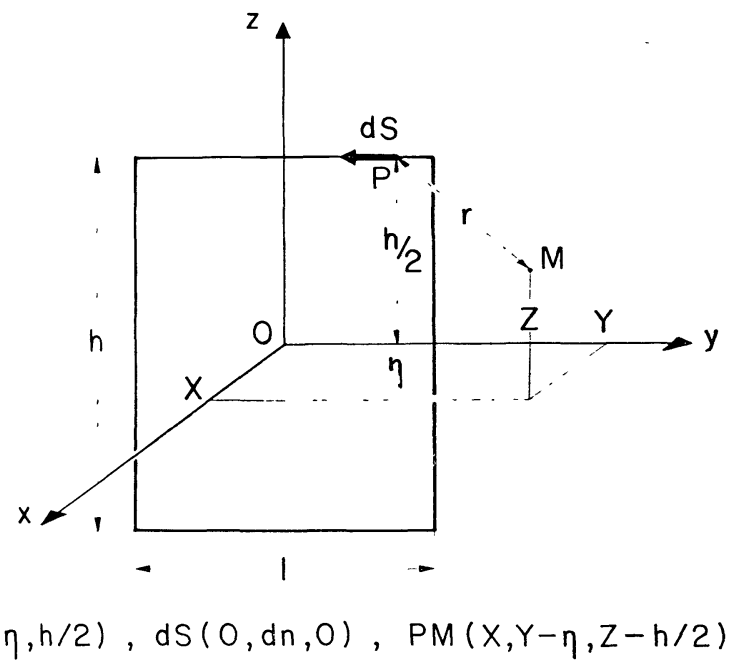

FIG. 6. 
(coordonnées du point ramenées au système d'axes défini par le cadre)

$$
\begin{aligned}
y_{1} & =y-\frac{l}{2} \\
y_{2} & =y+\frac{l}{2} \\
z_{1} & =z-\frac{h}{2} \\
z_{2} & =z+\frac{h}{2} \\
(a, b, c) & =\varphi(a, b)-\varphi(b, c) \\
\varphi(u, v) & =\frac{u}{\left(v^{2}+x^{2}\right)\left(u^{2}+v^{2}+x^{2}\right)^{1 / 2}} .
\end{aligned}
$$$$
\psi(a, b, c)=\varphi(a, b)-\varphi(b, c)
$$

On obtient :

$$
\begin{aligned}
& H X=H_{n} \cos \alpha+H_{h} \sin \alpha \\
& H Y=H_{n} \sin \alpha+H_{h} \cos \alpha \\
& H Z=H_{z} .
\end{aligned}
$$

Justification. - Il suffit de justifier la formule pour le cas où le centre de gravité du cadre est à l'origine $(Z C=0)$, où le plan du cadre coïncide avec le plan yoz $(C A=\alpha=0)$ et pour un cadre constitué par une seule spire $C N=1$.

La formule de Laplace nous donne le champ dH produit par un élément de courant ds

$$
\mathbf{d H}=\frac{i}{10} \frac{\mathbf{d s} \wedge \mathbf{P M}}{r^{3}} .
$$

Le courant est dirigé comme l'indique la figure 6 si l'on veut que $\mathrm{O} x$ soit la normale positive

$$
\begin{gathered}
\mathbf{d H}=\frac{i}{10}\left\{\begin{array}{l}
\operatorname{sur} \mathrm{O} x: \frac{(z-h / 2) \mathrm{d} \eta}{\left[x^{2}+(y-\eta)^{2}+(z-h / 2)^{2}\right]^{3 / 2}} \\
\operatorname{sur} \mathrm{O} y: 0 \\
\operatorname{sur} \mathrm{O} z: \frac{-x \mathrm{~d} \eta}{\left[x^{2}+(y-\eta)^{2}+(z-h / 2)^{2}\right]^{3 / 2}}
\end{array}\right. \\
\mathbf{H}_{\text {côté supérieur }}=\int_{\eta=+l / 2}^{\eta=-l / 2} \mathbf{d H}=\frac{i}{10}\left\{\begin{array}{l}
\operatorname{sur} \mathrm{O} x:\left|\frac{(z-h / 2)(y-\eta)}{\left[x^{2}+(z-h / 2)^{2}\right]\left[x^{2}+(y-\eta)^{2}+(z-h / 2)^{2}\right]^{1 / 2}}\right|_{\eta=-l / 2}^{\eta=+l / 2} \\
\operatorname{sur} \mathrm{O} y: 0 \quad \frac{-x(y-\eta)}{\operatorname{sur} \mathrm{O} z:\left|\frac{1}{\left[x^{2}+(z-h / 2)^{2}\right]\left[x^{2}+(y-\eta)^{2}+(z-h / 2)^{2}\right]^{1 / 2}}\right|_{\eta=-l / 2}^{\eta=+l / 2}}
\end{array}\right.
\end{gathered}
$$

d'après la formule (II) de l'annexe.

En utilisant les notations précédentes, on a :

$$
\mathbf{H}_{\text {côté superieur }}=\frac{i}{10} \mid \begin{aligned}
& z_{1} \psi\left(y_{1}, y_{2}, z_{1}\right) \\
& \vdots \quad \psi\left(y_{1}, y_{2}, z_{1}\right) .
\end{aligned} .
$$

On opérerait de même pour les autres côtés.

L'expression du champ total ressort du tableau suivant :

$$
\mathbf{H}=\frac{i}{10}\left\{\begin{array}{ccccc}
\text { Côté supérieur } & \text { Côté inférieur } & \text { Côté droit } & \text { Côté gauche } \\
\operatorname{sur} \mathrm{O} x & z_{1} \psi\left(y_{1}, y_{2}, z_{1}\right) & -z_{2} \psi\left(y_{1}, y_{2}, z_{2}\right) & y_{1} \psi\left(z_{1}, z_{2}, y_{1}\right) & -y_{2} \psi\left(z_{1}, z_{2}, y_{2}\right) \\
\operatorname{sur} \mathrm{O} y & 0 & 0 & -x \psi\left(z_{1}, z_{2}, y_{1}\right) & x \psi\left(z_{1}, z_{2}, y_{2}\right) \\
\operatorname{sur} \mathrm{O} z & -x \psi\left(y_{1}, y_{2}, z_{1}\right) & x \psi\left(y_{1}, y_{2}, z_{2}\right) & 0 & 0
\end{array}\right.
$$

Nous retrouvons bien les formules données au début de ce chapitre.

\section{SOUS-PROGRAMME FLUXA}

But. - Le sous-programme FLUXA permet de calculer le flux magnétique créé par un aimant élémen taire et traversant un cadre rectangulaire mince, de centre $\mathrm{O}$ et de normale orientée selon l'axe $\mathrm{O} x$ en fonction des données suivantes (Fig. 7) :

- XA, YA, ZA coordonnées $x_{\mathrm{A}}, y_{\mathrm{A}}, z_{\mathrm{A}}$ de l'aimant $(\mathrm{cm})$. 


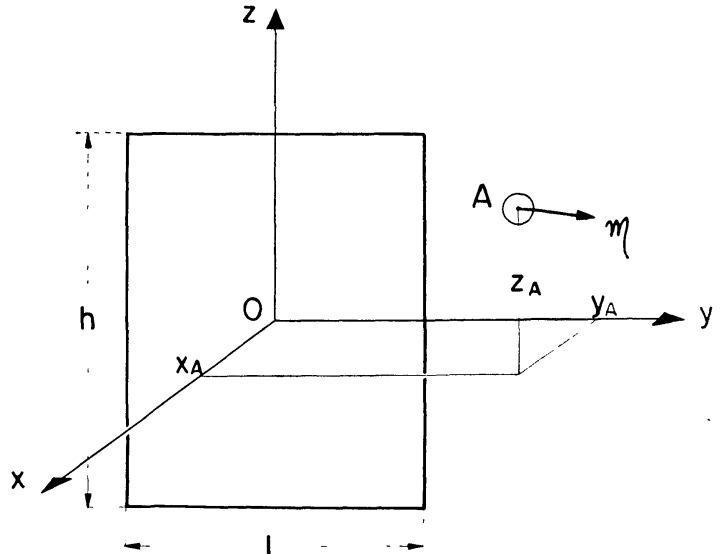

Fig. 7. - Le sous-programme FLUXA calcule le flux magnétique créé par un aimant é'émentaire à travers un cadre rectangulaire mince de centre $\mathrm{O}$ et de normale orientée selon l'axe $\mathrm{O} x$.

- CMX, CMY, CMZ composantes $\mathcal{N}_{x}, \mathcal{H}_{y}, \mathcal{N}_{z} \mathrm{du}$ moment magnétique de l'aimant (œrsted $/ \mathrm{cm}^{3}$ ).

- $\mathrm{CH}$ hauteur $h$ du cadre.

- CL largeur $l$ du cadre.

- CN nombre de spires.

Le flux magnétique PHI créé par l'aimant est exprimé en maxwells.

Formulation. - Le flux magnétique créé par un aimant élémentaire et traversant un cadre rectangulaire mince de centre $\mathrm{O}$ et situé dans le plan zoy est donné par:

$$
\begin{array}{r}
\mathrm{PHI}=\left[\Omega\left(a_{1}, b_{1}\right)-\Omega\left(a_{1}, b_{2}\right)-\Omega\left(a_{2}, b_{1}\right)+\right. \\
\left.+\Omega\left(a_{2}, b_{2}\right)\right] . \mathrm{CN}
\end{array}
$$

avec

$$
\begin{aligned}
\Omega(u, v)=\left\{\frac{u\left(v \mathcal{H}_{x}+x_{\mathrm{A}} \mathcal{M}_{z}\right)}{\left(x_{\mathrm{A}}^{2}+v^{2}\right)}+\right. & \left.\frac{v\left(u \mathcal{M}_{x}+x_{\mathrm{A}} \mathcal{M}_{y}\right)}{\left(x_{\mathrm{A}}^{2}+u^{2}\right)}\right\} \times \\
& \times \frac{1}{\left(x_{\mathrm{A}}^{2}+u^{2}+v^{2}\right)^{1 / 2}}
\end{aligned}
$$

où

$$
\begin{aligned}
& a_{1}=\frac{l}{2}-y_{\mathrm{A}}, \\
& a_{2}=-\frac{l}{2}-y_{\mathrm{A}}, \\
& b_{1}=\frac{h}{2}-z_{\mathrm{A}}, \\
& b_{2}=-\frac{h}{2}-z_{\mathrm{A}} .
\end{aligned}
$$

Démonstration. - 1. CHAMP CRÉÉ EN UN POINT M PAR UN AIMANT Élémentaire A (Fig. 8). - Soient :

$A$ l'aimant.

$\mathcal{H}$ son moment magnétique.

$\mathrm{M}$ le point où l'on calcule le champ.

He le champ créé.

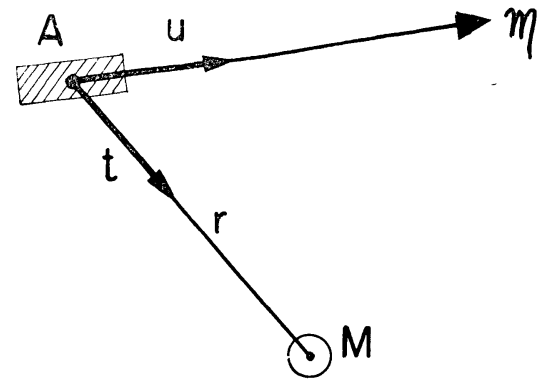

FIG. 8.

$\mathbf{u}$ vecteur unitaire selon $\mathcal{M}$,

t vecteur unitaire selon AM.

$r$ distance AM.

L'aimant $A$ peut être assimilé à deux masses magnétiques $-m$ et $+m$, de distance $\mathrm{d} A$ infiniment petite, alignées selon $\mathcal{M}$ avec :

$$
\mathcal{M}=m \mathbf{d A} \text {. }
$$

Le champ (répulsif) créé par la masse $+m$ supposée en $A$ est égal à :

$$
m \frac{\mathbf{t}}{r^{2}}=m \frac{r \mathbf{t}}{r^{3}}
$$

Le champ créé par l'aimant est égal au champ créé par la masse $(+m)$ moins le champ créé par la masse $(-m)$ :

$$
\mathscr{H}_{\mathrm{M}}=\mathrm{d}\left(\frac{m r \mathbf{t}}{r^{3}}\right)=m \mathrm{~d}\left(\frac{r \mathbf{t}}{r^{3}}\right)-3 m \frac{r \mathbf{t} \mathrm{d} r}{r^{4}},
$$

or

$$
\begin{aligned}
& r \mathbf{t}=\mathbf{A M} \quad \text { donc } \mathrm{d}(r \mathbf{t})=-\mathrm{d} \mathbf{A}=-\mathbf{u} \frac{\mathcal{M}}{m}, \\
& r^{2}=\mathbf{A M} \cdot \mathbf{A M} \quad \text { donc } \quad r \mathrm{~d} r=-r \mathbf{t u} \frac{\mathcal{M}}{m} .
\end{aligned}
$$

d'où

$$
\mathscr{H}_{\mathrm{M}}=\frac{\mathscr{N}}{r^{3}}(-\mathbf{u}+3 \mathbf{t}(\mathbf{t} \cdot \mathbf{u})) .
$$

2. FluX a tRaVers un ÉlÉment DE SURFACE d $S$ du CADRE RECTANGULAIRE (Fig. 9). - Si n est la normale au cadre, le flux à travers un élément de surface $\mathrm{d} S$ est égal à :

$$
\mathrm{d} \Phi=\mathscr{H}_{\mathrm{M}} \mathbf{n} \mathrm{d} S .
$$

Puisque le cadre est dans le plan yoz, n est parallèle à $\mathrm{O} x$ :

$$
\mathrm{d} \Phi=\mathcal{H}_{x} \mathrm{~d} S .
$$

Les coordonnées de l'aimant $A$ sont $x_{\mathrm{A}}, y_{\mathrm{A}}, z_{\mathrm{A}}$. 


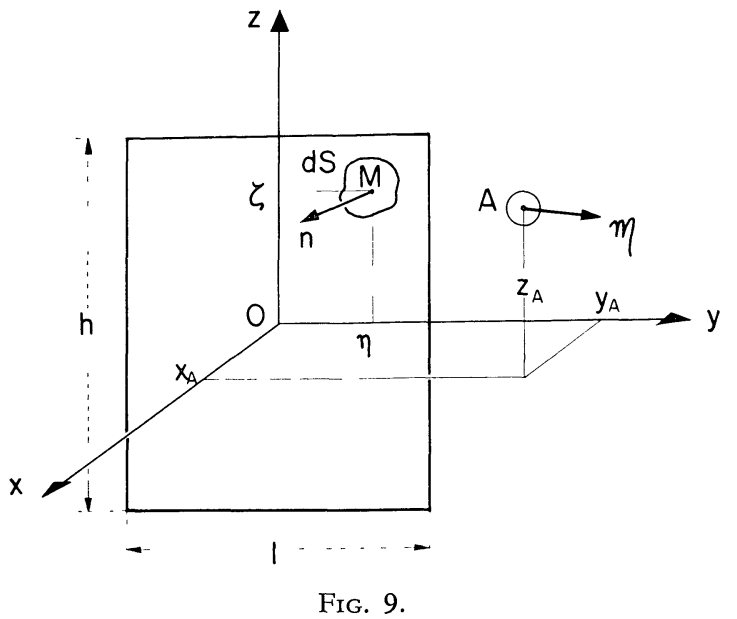

Les composantes du vecteur $u$ sont $\left(\mathcal{M}_{x} / \mathcal{M}, \mathcal{M}_{y} / \mathcal{M}\right.$, $\left.\mathcal{M}_{z} / \mathcal{M}\right)$ avec

$$
\mathcal{M}^{2}=\mathcal{M}_{x}^{2}+\mathcal{M}_{y}^{2}+\mathcal{M}_{z}^{2} .
$$

Les coordonnées d'un point $\mathrm{M}$ du cadre sont $(0, \eta, \zeta)$.

Les composantes du vecteur $\mathbf{t}$ sont

$$
\left(-x_{\mathrm{A}} / r,\left(\eta-y_{\mathrm{A}}\right) / r,\left(\zeta-z_{\mathrm{A}}\right) / r\right)
$$

avec

$$
r^{2}=x_{\mathrm{A}}^{2}+\left(\eta-y_{\mathrm{A}}\right)^{2}+\left(\zeta-z_{\mathrm{A}}\right)^{2} .
$$

Le flux créé à travers un élément de surface $\mathrm{d} S \mathrm{du}$ cadre rectangulaire est égal à :

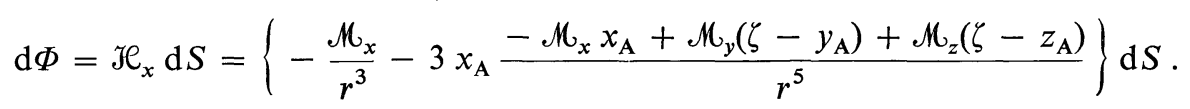

3. FluX a tRAVERS UNE SPIRE eNTière. - Le flux créé à travers une spire rectangulaire est égal à :

$$
\Phi=\int_{-h / 2}^{h / 2} \mathrm{~d} \zeta \int_{-l / 2}^{l / 2} \mathcal{H}_{x} \mathrm{~d} \eta .
$$

En posant $Y=\eta-y_{\mathrm{A}}, Z=\zeta-z_{\mathrm{A}}$ et en groupant convenablement les termes, on obtient:

$$
\Phi=\int_{Y=-l / 2-y_{\mathrm{A}}}^{l / 2-y_{\mathrm{A}}} \int_{Z=-h / 2-z_{\mathrm{A}}}^{h / 2-z_{\mathrm{A}}}\left[\mathcal{M}_{x} \frac{2 x_{\mathrm{A}}^{2}-Y^{2}-Z^{2}}{\left(x_{\mathrm{A}}^{2}+Y^{2}+Z^{2}\right)^{5 / 2}}-3 x_{\mathrm{A}} \frac{Y \mathcal{M}_{y}+Z \mathcal{M}_{z}}{\left(x_{\mathrm{A}}^{2}+Y^{2}+Z^{2}\right)^{5 / 2}}\right] \mathrm{d} Y \mathrm{~d} Z,
$$

ce qui donne après avoir utilisé les formules $(\mathrm{V})$ et $(\mathrm{VI})$ de l'annexe :

$$
\Phi=\left.\left|\frac{1}{\left(x_{\mathrm{A}}^{2}+Y^{2}+Z^{2}\right)^{1 / 2}}\left\{\frac{Y\left(\mathcal{H}_{x} Z+x_{\mathrm{A}} \mathcal{H}_{z}\right)}{x_{\mathrm{A}}^{2}+Z^{2}}+\frac{Z\left(\mathcal{H}_{x} Y+x_{\mathrm{A}} \mathcal{M}_{y}\right)}{x_{\mathrm{A}}^{2}+Y^{2}}\right\}\right|_{Z=-h / 2-z_{A}}^{h / 2-z_{A}}\right|_{Y=-l / 2-y_{A}} ^{l / 2-y_{A}}
$$

qui est la formule proposée dans le paragraphe formulation.

\section{SOUS-PROGRAMME FLUXB}

But. - Le sous-programme FLUXB permet de calculer le flux magnétique créé par un aimant élémentaire à travers un cadre rectangulaire infiniment mince de position quelconque en fonction des données suivantes (Fig. 10) :

- A (1 à 3) coordonnées $x_{\mathrm{A}}, y_{\mathrm{A}}, z_{\mathrm{A}}$ de l'aimant (cm).

- CM (1 à 3) composantes $\mathcal{M}_{x}, \mathcal{M}_{y}, \mathcal{M}_{z}$ du moment magnétique de l'aimant (œrsted $/ \mathrm{cm}^{3}$ ).

- C (1 à 3) coordonnées $x_{\mathrm{c}}, y_{\mathrm{c}}, z_{\mathrm{c}}$ du centre $\Omega \mathrm{du}$ cadre $(\mathrm{cm})$.

- CH (1 à 3) projections $h_{x}, h_{y}, h_{z}$ de l'un des côtés du cadre $(\mathrm{cm})$.

- CL (1 à 3) projections $l_{x}, l_{y}, l_{z}$ d'un deuxième côté du cadre $(\mathrm{cm})$. Les vecteurs $\mathrm{CH}$ et $\mathrm{CL}$ doivent être orthogonaux.

- DN direction de la normale $n$ orientée au cadre : $\mathrm{DN}=+1$ si $n$ a même sens que le produit vectoriel

$$
\mathbf{C L} \wedge \mathbf{C H} \text {, }
$$

$\mathrm{DN}=-1$ si $n$ a le sens opposé.

- $\mathrm{CN}$ nombre de spires.

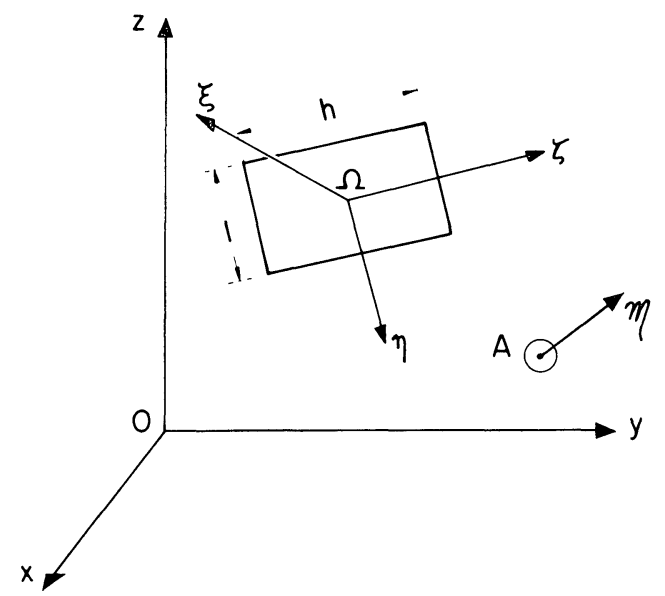

FIG. 10. - Le sous-programme FLUXB calcule le flux magnétique créé par un aimant élémentaire à travers un cadre rectangulaire mince de position quelconque. 
Le flux magnétique PHI créé par l'aimant est mesuré en maxwells.

Formulation. - Nous cherchons un changement d'axes qui fasse correspondre le trièdre de référence $(\mathrm{O} x y z)$ au trièdre lié au cadre $(\Omega \xi \eta \zeta)$ de façon à utiliser le sous-programme FLUXA avec des données que nous allons déterminer. Ce changement d'axes amène :

- l'origine $\mathrm{O}$ au centre du cadre $\left(x_{\mathrm{c}}, y_{\mathrm{c}}, z_{\mathrm{c}}\right)$,

- l'axe $\Omega \eta$ parallèle à $\mathrm{CL}$,

- l'axe $\Omega \zeta$ parallèle à $\mathrm{CH}$ si $\mathrm{DN}=+1$,

- l'axe $\Omega \zeta$ antiparallèle à $\mathrm{CH}$ si $\mathrm{DN}=-1$,

- l'axe $\Omega \xi$ formant un trièdre direct avec $\Omega \eta$ et $\Omega \zeta$.

Cette transformation de coordonnées s'écrit :

$$
\left\{\begin{array}{l}
\xi=\alpha\left(x-x_{\mathrm{c}}\right)+\alpha^{\prime}\left(y-y_{\mathrm{c}}\right)+\alpha^{\prime \prime}\left(z-z_{\mathrm{c}}\right) \\
\eta=\beta\left(x-x_{\mathrm{c}}\right)+\beta^{\prime}\left(y-y_{\mathrm{c}}\right)+\beta^{\prime \prime}\left(z-z_{\mathrm{c}}\right) \\
\zeta=\gamma\left(x-x_{\mathrm{c}}\right)+\gamma^{\prime}\left(y-y_{\mathrm{c}}\right)+\gamma^{\prime \prime}\left(z-z_{\mathrm{c}}\right)
\end{array}\right.
$$

ou

$$
\left\{\begin{array}{l}
x=x_{\mathrm{c}}+\alpha \xi+\beta \eta+\gamma \zeta \\
y=y_{\mathrm{c}}+\alpha^{\prime} \xi+\beta^{\prime} \eta+\gamma^{\prime} \zeta \\
z=y_{\mathrm{c}}+\alpha^{\prime \prime} \check{\zeta}+\beta^{\prime \prime} \eta+\gamma^{\prime \prime} \zeta
\end{array}\right.
$$

avec :

1) CL vecteur dirigé selon $\boldsymbol{\Omega} \boldsymbol{\eta}$, on a donc :

$$
\beta=\frac{l_{x}}{l}, \beta^{\prime}=\frac{l_{y}}{l}, \beta^{\prime \prime}=\frac{l_{z}}{l}\left(l^{2}=l_{x}^{2}+l_{y}^{2}+l_{z}^{2}\right) .
$$

2) $\mathrm{CH}$ vecteur dirigé selon $\pm \Omega \zeta$, le signe étant défini par $\mathrm{DN}$ :

$$
\gamma=\delta \frac{h_{x}}{h}, \gamma^{\prime}=\delta \frac{h_{y}}{h}, \gamma^{\prime \prime}=\delta \frac{h_{z}}{h}
$$

avec $\delta=$ signe de DN et $h^{2}=h_{x}^{2}+h_{y}^{2}+h_{z}^{2}$.

3) Le trièdre $\left(\Omega \xi_{\eta} \zeta\right)$ doit être direct, on a donc :

$$
\begin{aligned}
\alpha & =\beta^{\prime} \gamma^{\prime \prime}-\gamma^{\prime} \beta^{\prime \prime} \\
\alpha^{\prime} & =\beta^{\prime \prime} \gamma-\gamma^{\prime \prime} \beta \\
\alpha^{\prime \prime} & =\beta \gamma^{\prime}-\gamma \beta^{\prime} .
\end{aligned}
$$

Ce changement d'axes étant opéré, pour obtenir le flux magnétique créé par un aimant à travers un cadre rectangulaire de position quelconque, il suffit d'utiliser le sous-programme FLUXA avec les paramètres suivants :

$$
\begin{aligned}
\mathrm{XA} & =\alpha\left(x_{\mathrm{A}}-x_{\mathrm{c}}\right)+\alpha^{\prime}\left(y_{\mathrm{A}}-y_{\mathrm{c}}\right)+\alpha^{\prime \prime}\left(z_{\mathrm{A}}-z_{\mathrm{c}}\right) \\
\mathrm{YA} & =\beta\left(x_{\mathrm{A}}-x_{\mathrm{c}}\right)+\beta^{\prime}\left(y_{\mathrm{A}}-y_{\mathrm{c}}\right)+\beta^{\prime \prime}\left(z_{\mathrm{A}}-z_{\mathrm{c}}\right) \\
\mathrm{ZA} & =\gamma\left(x_{\mathrm{A}}-x_{\mathrm{c}}\right)+\gamma^{\prime}\left(y_{\mathrm{A}}-y_{\mathrm{c}}\right)+\gamma^{\prime \prime}\left(z_{\mathrm{A}}-z_{\mathrm{c}}\right) \\
\mathrm{CMX} & =\alpha \mathcal{M}_{x}+\alpha^{\prime} \mathcal{H}_{y}+\alpha^{\prime \prime} \mathcal{M}_{z} \\
\mathrm{CMY} & =\beta \mathcal{H}_{x}+\beta^{\prime} \mathcal{M}_{y}+\beta^{\prime \prime} \mathcal{M}_{z} \\
\mathrm{CMZ} & =\gamma \mathcal{H}_{x}+\gamma^{\prime} \mathcal{M}_{y}+\gamma^{\prime \prime} \mathcal{M}_{z} \\
\mathrm{CH} & =h \\
\mathrm{CL} & =l .
\end{aligned}
$$

Le sous-programme FLUXB calcule ces quantités et les introduit dans le sous-programme FLUXA pour déterminer le flux $\Phi$.

\section{SOUS-PROGRAMMES DE SERVICE}

Nous donnons ci-dessous un descriptif très bref des sous-prọgrammes de service utilisés lors des calculs de champ magnétique. Ces sous-programmes ont été écrits et mis au point pour être utilisés sur l'ordinateur Control Data CDC 6600.

Sous-programme INTEGR. - C'est un sous-programme mathématique à caractère général de quadrature numérique permettant de calculer toute intégrale définie :

$$
S=\int_{a}^{b} f(x) \mathrm{d} x
$$

avec la précision que l'on veut.

Le calcul s'effectue par la méthode de Simpson et utilise la technique du pas variable automatique dans le double but de limiter les calculs tout en respectant la précision demandée par l'utilisateur. Lorsque cette précision n'a pu-être atteinte, le sous-programme prévient l'utilisateur en imprimant un diagnostic.

Les commentaires placés au début du programme en précisent le mode d'emploi.

Fonctions ECF et ECE. - Ces fonctions sont également mathématiques et à caractère général. Elles calculent les intégrales elliptiques complètes de première et de seconde espèce :

$$
\begin{aligned}
& \operatorname{ECF}(x)=\int_{0}^{\pi / 2} \frac{\mathrm{d} \varphi}{\sqrt{1-x^{2} \sin ^{2} \varphi}} \\
& \operatorname{ECE}(x)=\int_{0}^{\pi / 2} \sqrt{1-x^{2} \sin ^{2} \varphi} \mathrm{d} \varphi .
\end{aligned}
$$

Le calcul s'effectue par le biais des moyennes arithmético-géométriques.

3. Mode d'utilisation de ces différents sous-programmes. - a) Pour calculer le champ magnétique créé par une bobine épaisse d'axe vertical en un point extérieur à cette bobine, on utilise le sous-programme BOB.

b) Pour calculer le champ magnétique créé par une bobine mince d'axe vertical en un point intérieur ou extérieur à cette bobine, on utilise BOB2 qui lui-même fait appel à des sous-programmes de service INTEGR, $\mathrm{ECF}, \mathrm{ECE}$ et $\mathrm{F} 1$.

c) Pour calculer le champ magnétique créé par un cadre mince en un point, on utilise le sous-programme HCADRE.

d) Pour calculer le flux magnétique créé par un aimant élémentaire à travers un cadre rectangulaire mince, de centre $\mathrm{O}$ et de normale orientée selon l'axe $\mathrm{O} x$, on utilise le sous-programme FLUXA.

e) Pour calculer le flux magnétique créé par un aimant élémentaire à travers un cadre rectangulaire 
de position quelconque, on utilise le sous-programme FLUXB qui lui-même fait appel au sous-programme FLUXA.

4. Graphiques des lignes de force et des lignes d'égale valeur du champ magnétique créé par une bobine circulaire mince. - A titre d'illustration, nous présentons deux graphiques relatifs au champ créé par une bobine circulaire infiniment mince. Ces graphiques ont été obtenus sur traceur CALCOMP piloté par bande magnétique à partir des calculs effectués sur l'ordinateur Control Data CDC 6600.

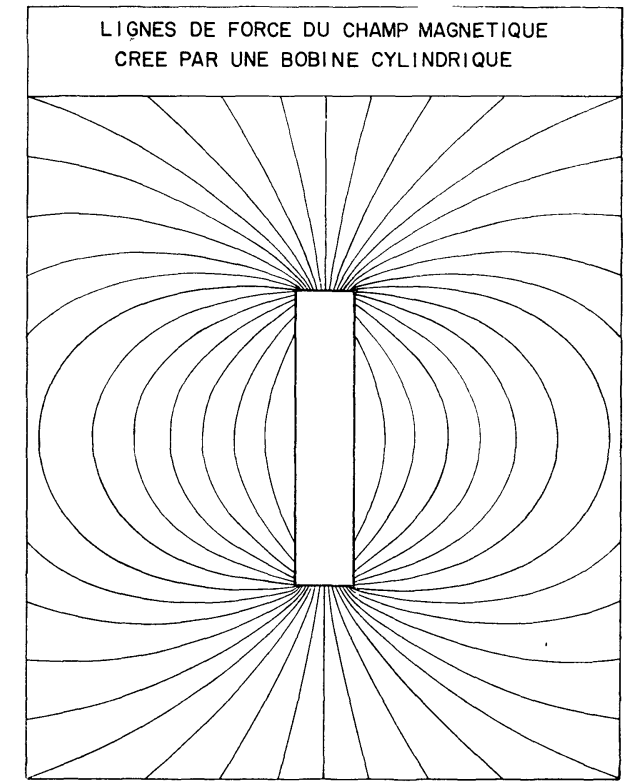

FIG. 11.

Le premier graphique (Fig. 11) donne un schéma des lignes de force. Il fait bien ressortir l'aspect canalisé de celles-ci à l'intérieur de la bobine et leur épanouissement dans toutes les directions à la sortie de la bobine.

Il est à noter que des lignes de force viennent buter sur les flancs de la bobine. En fait, elles traversent cette dernière en subissant une "réfraction». Ce résultat est classique et traduit le fait qu'à la traversée de la bobine la composante verticale change de $4 \pi \sigma$.

Le deuxième graphique (Fig. 12) donne un schéma

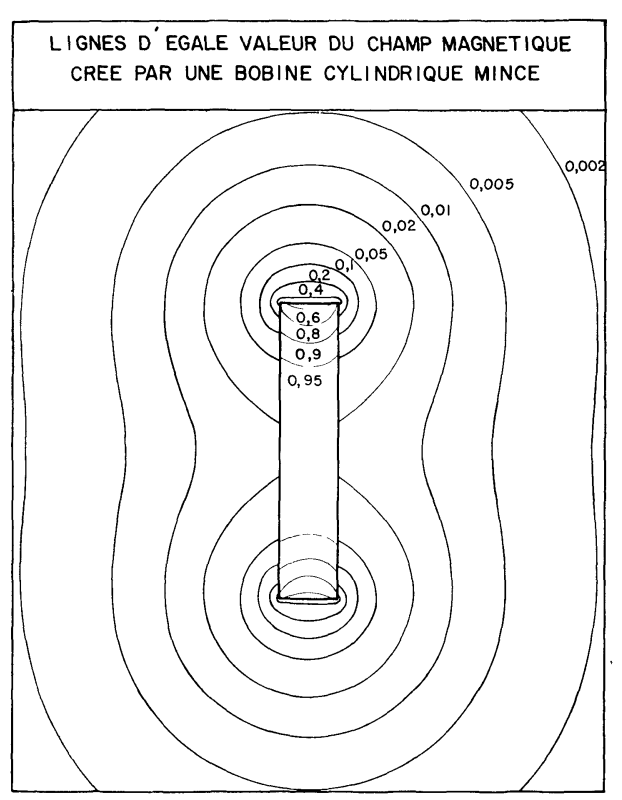

FIG. 12.

des lignes d'égale valeur du champ. Les graduations des courbes sont relatives, le champ étant pris égal à l'unité au centre de la bobine. On remarquera, ce qui est également classique, que les valeurs du champ sont fortes à l'intérieur de la bobine et décroissent rapidement à l'extérieur. Certaines des courbes tracées viennent buter sur la bobine : elles y marquent un point d'arrêt et ne la traversent pas.

Remerciements. - Les auteurs remercient Madame Baudet et Mademoiselle Roy pour leur contribution à cette étude.

Remarque. - Les textes FORTRAN des sousprogrammes peuvent être obtenus sur simple demande au Journal de Physique.

\section{ANNEXE}

\section{Formules mathématiques}

$$
\begin{aligned}
& \int \frac{z \mathrm{~d} z}{\left(a^{2}+z^{2}\right)^{3 / 2}}=\frac{-1}{\sqrt{a^{2}+z^{2}}} . \\
& \int \frac{\mathrm{d} z}{\left(a^{2}+z^{2}\right)^{3 / 2}}=\frac{z}{a^{2} \sqrt{a^{2}+z^{2}}} . \\
& \int \frac{z \mathrm{~d} z}{\left(a^{2}+z^{2}\right)^{5 / 2}}=\frac{-1}{3\left(a^{2}+z^{2}\right)^{3 / 2}} . \\
& \int \frac{\mathrm{d} z}{\left(a^{2}+z^{2}\right)^{5 / 2}}=\frac{z\left(2 z^{2}+3 a^{2}\right)}{3 a^{4}\left(a^{2}+z^{2}\right)^{3 / 2}} .
\end{aligned}
$$




$$
\begin{gathered}
\iint \frac{u \mathrm{~d} u \mathrm{~d} v}{\left(a^{2}+u^{2}+v^{2}\right)^{5 / 2}}=-\frac{v}{3\left(a^{2}+u^{2}\right) \sqrt{a^{2}+u^{2}+v^{2}}}+f(u)+g(v)\left(^{*}\right) . \\
\iint \frac{2 a^{2}-u^{2}-v^{2}}{\left(a^{2}+u^{2}+v^{2}\right)^{5 / 2}} \mathrm{~d} u \mathrm{~d} v=\frac{u v}{\sqrt{a^{2}+u^{2}+v^{2}}}\left(\frac{1}{a^{2}+u^{2}}+\frac{1}{a^{2}+v^{2}}\right)+f(u)+g(v)\left(^{*}\right) . \\
F(x)=\int_{0}^{\pi / 2} \frac{\mathrm{d} \varphi}{\sqrt{1-x^{2} \sin ^{2} \varphi}}=\text { intégrale elliptique complète de première espèce. } \\
E(x)=\int_{0}^{\pi / 2} \sqrt{1-x^{2} \sin ^{2} \varphi} \mathrm{d} \varphi=\text { intégrale elliptique complète de deuxième espèce. } \\
G(x)=\frac{F(x)-E(x)}{x^{2}} \quad \operatorname{remarque:} \quad G(0)=\frac{\pi}{4} . \\
\int_{0}^{2 \pi} \frac{\cos \alpha \mathrm{d} \alpha}{\sqrt{a-b \cos \alpha}}=\frac{4}{\sqrt{a+b}}[2 G(\lambda)-F(\lambda)] \quad \text { avec } \quad \lambda=\sqrt{\frac{2 b}{a+b}} \quad \text { et } \quad 0 \leqslant b<a .
\end{gathered}
$$

Cette formule se démontre par le changement de variable $\varphi=(\pi-\alpha) / 2$.

$\left(^{*}\right)$ Les fonctions $f$ et $g$ sont arbitraires. Elles s'éliminent lorsque l'intégration s'effectue dans un domaine rectangulaire dont les côtés sont parallèles aux axes.

\section{Bibliographie}

FRISCHKNECHT (F. C.), Quaterly of the Colorado School of Mines, 1967, 1 .

HAMming (R. W.), Numerical methods for scientists and engineers, 1962, 411.

Wait (J. R.), Can. J. Phys., 1951, 6, 577.
WAIT (J. R.), Geophysics, 1954, 2, 290.

WAIT (J. R.), Geophysics, 1955, 3, 630.

WAIT (J. R.), Geophysics, 1956, 2, 449.

WaIt (J. R.), Appl. Sci. Research, 1958, 78.

WAIT (J. R.), Geophysics, 1962, 3, 382. 